\title{
Technology and Pregnancy
}

\author{
Adrian Cotarelo ${ }^{1 *}$, Homaira T. Zaman ${ }^{1 *}$, Lois Jovanovič ${ }^{1}$, and Moshe Hod ${ }^{2}$
}

\section{Introduction}

I N PART BECAUSE OF THE GLOBAL OBESITY EPIDEMIC, pregnancies complicated by diabetes have grown into a widespread issue throughout all parts of the world. There are over 25 million people affected by diabetes in the United States alone, with 12.6 million diabetic women aged 20 and over (1). Maternal hyperglycemia can have devastating effects on pregnancy when uncontrolled, including above-average birth weight, macrosomia, birth defects, and an increased risk for obesity and diabetes in the future. This year, we reviewed 1,299 articles and came to a final listing of 81 possible articles to analyze. We selected 15 studies that we felt were representative of the research conducted in the field of technology, treatment, and pregnancy over the course of the past year. A large proportion of these original articles related to diagnostic methods for determining gestational diabetes mellitus (GDM). While this topic comprised much of the literature over the past year, we chose to address this topic by citing the National Institutes of Health (NIH) consensus article on the matter. Studies were reviewed systematically and evaluated for sample size, implications on diabetes care, and the validity of their findings.

\section{Gestational diabetes, pre-pregnancy obesity and pregnancy weight gain in relation to excess fetal growth: variations by race/ethnicity}

Bowers $K^{1,2}$, Laughon $S K^{1}$, Kiely $M^{1}$, Brite $J^{3}$, Chen $Z^{4}$, Zhang $C^{1}$

${ }^{1}$ Epidemiology Branch and ${ }^{4}$ Biostatistics Branch, Division of Epidemiology, Statistics, and Preventive Research, Eunice Kennedy Shriver National Institute of Child Health and Human Development, Rockville, MD; ${ }^{2}$ Present address: Division of Biostatistics and Epidemiology, Cincinnati Children's Hospital Medical Center, Cincinnati, OH; and ${ }^{3} \mathrm{CUNY}$ Institute of Demographic Research and CUNY School of Public Health at Hunter College City University of New York, New York, NY

Diabetologia 2013; 56: 1263-71

\section{Objective}

The incidence of pediatric obesity and diabetes is increasing throughout the world, with children of ethnic backgrounds being impacted more severely. This study observed the effects of prepregnancy adiposity, weight gain during the pregnancy, and GDM on excess fetal growth to evaluate the susceptibility of various ethnic populations to adverse effects of hyperglycemia.

\section{Methods}

A retrospective observational study was formed from a cohort of 228,562 births between 2002 and 2008 across 19 hospitals spread throughout the United States. Ethnicities and races observed included non-Hispanic white, non-Hispanic black, Hispanic, and Asian/Pacific Islander. Data were abstracted from electronic medical records. Exclusion criteria included multiple gestation pregnancies, and limited available data on relevant statistics such as GDM, prepregnancy body-mass index (BMI), and birth weight. The final cohort consisted of 105,985 pregnancies. High pregnancy weight gain was defined as that which was greater than the median weight gain observed in the cohort. Each of three factors (prepregnancy adiposity, weight gain during the pregnancy, and GDM) were evaluated independently for association with large-for-gestational-age (LGA) and macrosomic infants. Each was then weighed together in order to observe the joint effects between the presence of one, two, or all three factors.

\section{Results}

All three factors were found to be independently associated with an increased risk for LGA births. This was true for all races with the exception of non-Hispanic white women, who did not observe an increase in LGA births from GDM alone. 10,689 neonates were LGA, and 8,682 were macrosomic. In general, LGA and macrosomic infants were more likely to be born to women with GDM, as well as those who had a previous macrosomic infant. Birth weight was linearly associated with prepregnancy BMI, with the highest proportion of LGA

\footnotetext{
${ }^{1}$ Sansum Diabetes Research Institute, Santa Barbara, CA.

${ }^{2}$ Helen Schneider Hospital for Women, Rabin Medical Center, Petah Tikva, Israel.

*These two authors contributed equally to this work.
} 
and macrosomic infants born to mothers with a prepregnancy BMI over $30 \mathrm{~kg} / \mathrm{m}^{2}$. Asian and Pacific Islander (PI) women had the highest percentage of LGA infants and GDM, while white non-Hispanic women had the lowest. Asian and PI women were also less likely to be overweight or obese before the pregnancy. A significant interaction was observed between race, BMI, and GDM incidence. With the exception of nonHispanic white women with GDM, the presence of any of the observed factors was significantly associated with LGA infants. The presence of two factors increased the risk further across all races. The presence of all three did not increase the risk by a significant amount further in Asian women, while they nearly doubled the risk for Hispanic and non-Hispanic white women compared with being exposed to only two factors.

\section{Conclusions}

GDM, prepregnancy adiposity, and pregnancy weight gain were each associated with an increased birth weight, while combinations of these factors increased the risk for LGA and macrosomia even further. The degree to which these combinations of factors affected the risk for LGA and macrosomic births depended on race. This may be a genetic difference or due to physiological differences in fat storage or glucose metabolism between races. Due to differences in body size between races and genders, it is not appropriate in a clinical setting to identify a general cut-off weight for LGA and macrosomia. Race must also be considered due to these differences in genetics and physiology. GDM was greatly associated with an increased risk of an LGA or macrosomic birth, including those who were underweight and of normal weight. This increase in risk varied by race, prepregnancy BMI, and pregnancy weight gain, with an increased joint effect that varied by race. A woman's race should be considered when evaluating the prevention strategy for excess fetal growth in a pregnancy complicated by GDM.

\section{Comment}

This study had an impressively large and varied sample size from throughout the United States, granting their result's generalizability. One limitation addressed in the article is the combination of Asian and Pacific Islander races. These were factored in together because of the organization of the Consortium of Safe Labor database, which provided the patient data for the study. Differences in risk for LGA and macrosomia have been noted between Asian and Pacific Islander women, and this may have affected the findings relating to this group. However, the study makes a strong case for a further consideration of race among women with GDM. The observation of a synergistic effect of the three additional factors evaluated by the study allows for a more aggressive treatment plan for women presenting with the relevant risk factors for their race and should be considered in future GDM care.

\section{Association between serum 25-hydroxyvitamin D in early pregnancy and risk of gestational diabetes mellitus}

Parlea $L^{1}$, Bromberg $I L^{2,3}$, Feig $D S^{3-5}$, Vieth $R^{2,3,6}$, Merman $E^{1}$, Lipscombe $L L^{4,7}$
${ }^{1}$ Faculty of Medicine; ${ }^{2}$ Department of Laboratory Medicine and Pathobiology, Faculty of Medicine; ${ }^{4}$ Department of Medicine; and ${ }^{6}$ Department of Nutritional Sciences, University of Toronto, Toronto, Ontario, Canada; Departments of ${ }^{3}$ Pathology and Laboratory Medicine and ${ }^{5}$ Medicine, Mount Sinai Hospital, New York, NY; and ${ }^{7}$ Department of Medicine, Women's College Research Institute, Women's College Hospital, Toronto, Ontario, Canada

Diabet. Med. 2012; 29: e25-e32

\section{Vitamin D deficiency in pregnancy and gestational diabetes mellitus}

Burris $H H^{1,2}$, Rifas-Shiman $S L^{7}$, Kleinman $K^{7}$, Litonjua $A A^{4,5}$, Huh $S Y^{3}$, Rich-Edwards $J^{6,8}$, Camargo CA Jr. ${ }^{4,10}$, Gillman $M W^{7,9}$

${ }^{1}$ Department of Neonatology, Beth Israel Deaconess Medical Center, Boston, MA; Divisions of ${ }^{2}$ Newborn Medicine and ${ }^{3}$ Gastroenterology and Nutrition, Department of Pediatrics, Children's Hospital Boston, Boston, $M A ;{ }^{4}$ Channing Laboratory, ${ }^{5}$ Division of Pulmonary and Critical Care, and ${ }^{6}$ Division of Women's Health, Department of Medicine, Brigham \& Women's Hospital, Boston, MA; ${ }^{7}$ Obesity Prevention Program, Department of Population Medicine, Harvard Medical School, and Harvard Pilgrim Health Care Institute, Boston, MA; Departments of ${ }^{8}$ Epidemiology and ${ }^{9}$ Nutrition, Harvard School of Public Health, Boston, MA; and ${ }^{10}$ Department of Emergency Medicine, Massachusetts General Hospital, Harvard Medical School, Boston, MA

Am J Obstet Gynecol 2012; 207: 182.e1-8.

\section{Objective}

Both of the above studies aimed to evaluate whether or not low levels of 25-hydroxyvitamin D in pregnancy were associated with an increased risk of developing GDM. The Parlea study measured vitamin D levels in the first trimester between weeks 15 and 18, while the Burris study tested for vitamin $\mathrm{D}$ levels during the second trimester between weeks 26 and 28 .

\section{Methods}

The Parlea study was a nested case-control study that gathered data from March 1, 2008, to December 31, 2009, and 116 cases of women with GDM were compared with 219 control subjects. 25-Hydroxyvitamin D levels were obtained from antenatal blood samples. The Burris study was a prospective cohort of 1,314 women enrolled into the study before the 22nd week of their pregnancy. 25-Hydroxyvitamin D levels were measured during a GDM screening involving a 1hour $50 \mathrm{~g}$ glucose challenge test.

\section{Results}

In the Parlea study, GDM patients had significantly lower 25-hydroxyvitamin D levels in the first trimester than control subjects. Vitamin D levels below the top quartile $(<73.5 \mathrm{nmol} / \mathrm{L})$ were associated with a doubled likelihood of developing GDM. During the Burris study, patients with a vitamin D level below $25 \mathrm{nmol} / \mathrm{L}$ were associated with an increased risk for GDM. Both studies resulted in an odds ratio of 2.2 for increased risk of GDM when 25-hydroxyvitamin D levels were low. 


\section{Conclusions}

Both studies arrived at the conclusion that low 25hydroxyvitamin D levels resulted in a significantly increased risk for GDM. While they tested for vitamin D levels at different points in the pregnancy, the association with GDM was the same. As vitamin D may affect glucose tolerance, future treatment of GDM patients should monitor vitamin D levels in order to better care for pregnant patients.

\section{Comment}

The Burris study had a large sample size, adding to the validity of its results. While the Parlea study was smaller by almost 10 -fold, both analyses came to the same conclusions. As vitamin D levels appear to have a significant impact on the incidence of GDM, it may be worthwhile for providers to consider the findings of these studies for future care of pregnant patients already at risk for developing GDM.

\section{National institutes of health consensus development conference statement: diagnosing gestational diabetes mellitus, March 4-6, 2013}

Consensus Development Panel: VanDorsten ${ }^{1}$ et al.

${ }^{1}$ Division of Maternal-Fetal Medicine, Medical University of South Carolina, Charleston, SC

Obstet Gynecol 2013; 122: 358-69

\section{Objective}

At the present, the standard screening for GDM recommended by the American College of Obstetricians and Gynecologists involves first performing a glucose challenge test in which serum glucose is measured 1 hour after a woman drinks a $50 \mathrm{~g}$ glucose solution. If these results are abnormal, an oral glucose tolerance test (OGTT) is then performed. The OGTT requires that a woman's blood is drawn four times over 3 hours as she drinks a 100 g glucose solution. The International Association of the Diabetes and Pregnancy Study Groups (IADPSG) has proposed a one-step approach for GDM diagnosis involving a 2-hour OGTT with three blood sugar measurements: one fasting blood glucose, and one at the 1-hour and 2-hour marks. If any of the three are abnormal, GDM is diagnosed. This proposed method is estimated to at least double the number of GDM diagnoses in the United States. The purpose of this statement is to determine if, at this point in time, the NIH can recommend that the United States begin to adopt the onestep approach to diagnosing GDM.

\section{Methods}

The Eunice Kennedy Shriver National Institute of Child Health and Human Development, National Institute of Diabetes and Digestive and Kidney Diseases, National Institute of Nursing Research, Office of Research on Women's Health, Centers for Disease Control and Prevention, and the NIH Office of Disease Prevention convened to review the available scientific evidence in order to assess the official stance on GDM diagnoses. The panel included experts in many fields including, but not limited to, maternal and fetal medicine, healthcare economics, and obstetrics and gynecology. A planning committee developed seven questions to be addressed by the Consensus Development Panel.

\section{Considerations}

The panel evaluated current screening and diagnostic methods for GDM, the thresholds for these approaches, and how these thresholds were chosen. The impact of a one-step diagnostic tool for GDM on the U.S. healthcare system, as well as the health outcomes of undiagnosed women and their children, were assessed. Finally, the panel considered which diagnostic approach for GDM should be recommended, as well as any research gaps in the approach to GDM diagnosis.

\section{Conclusions}

The one-step approach offers some advantages over the current method, primarily used in the United States alone. There is value in adopting the international standard for GDM diagnosis in terms of both future research and outcome comparison between nations. Additionally, it would allow the diagnosis of GDM to be completed in one visit rather than two. There are concerns involving the large increase in the number of GDM diagnoses if the one-step method were implemented. There is also a lack of research to determine whether these additional GDM women would gain benefit from the treatment, and if it is worth the additional cost toward healthcare. The panel does not believe that there is sufficient evidence to adopt the one-step approach recommended by the IADPSG. Additional research is needed before the one-step approach can be recommended.

\section{Comment}

While the panel considered many effects that the conversion to the one-step approach will have on the United States, we feel that the one-step approach is entirely appropriate and should be adopted in the United States. While the one-step would increase the number of GDM diagnoses, and the associated costs involved with diagnosis and treatment, the United States is among the few that are not yet on board with this diagnostic method, calling into question the conclusion that further research is necessary.

\section{Maternal smoking during pregnancy and daughters' risk of gestational diabetes and obesity}

Mattsson $K^{1}$, Källén $K^{1}$, Longnecker $M P^{2}$, Rignell-Hydbom $A^{1}$, Rylander $L^{1}$

${ }^{1}$ Division of Occupational and Environmental Medicine, Institute of Laboratory Medicine, Lund University, Lund, Sweden; and ${ }^{2}$ Epidemiology Branch, National Institute of Environmental Health Sciences, NIH, DHHS, Research Triangle Park, NC

Diabetologia 2013; 56: 1689-95

\section{Objective}

This study aimed to examine the risk of developing GDM after being exposed to tobacco smoke in utero. Patients were also assessed for an increased risk of obesity or type 1 or type 2 diabetes mellitus. 


\section{Methods}

Data from 80,189 pregnancies were collected from the Medical Birth Register of Sweden. In utero tobacco exposure was classified as nonsmoker, moderate exposure (1-9 cigarettes/ day), and heavy exposure (10 cigarettes / day or more). Maternal smoking habits during the pregnancy were obtained via selfreport by trained midwives during their first antenatal visit. Data were collected for pregnancies that occurred from 1982 to 2010. These mothers were flagged as generation 1 (G1), and their children as generation 2 (G2). G2 women were observed for outcomes of GDM, nongestational diabetes, and obesity.

\section{Results}

The adjusted odds ratio for gestational diabetes and obesity was increased in those women who had been exposed both moderately and severely to in utero tobacco smoke. Women exposed to heavy tobacco smoke had a decreased adjusted odds ratio for nongestational diabetes. Obesity appeared to have a dose-response association with in utero tobacco exposure, with heavily exposed women having an increased risk over moderately exposed women.

\section{Conclusions}

Women who were exposed to tobacco smoke in utero are at a higher risk for developing gestational diabetes and obesity later in life. A decreased risk of nongestational diabetes was observed for women exposed heavily to tobacco in utero. This association remained even after adjusting for age, parity, BMI, gestational age, mode of delivery, birth weight, and age of the mother. While the majority of nongestational diabetes cases observed in the study were type 1 , this is similar to the prevalence of type 1 diabetes throughout Sweden.

\section{Comment}

Few studies are available on the effects of in utero tobacco exposure later in life. The information on smoking was obtained via self-report, which could contain a large source of error. People may not report honestly, particularly on a subject that may be taboo such as smoking habits during a pregnancy. The Sweden Medical Birth Register (MBR) has very high levels of completion to its data; however, the MBR does not record what week of gestation the first antenatal visit occurs. Therefore, it is not possible to tell if the meeting influenced smoking status for later parts of the pregnancy. The study concludes that heavy smoking during pregnancy decreases type 1 and type 2 diabetes later in life, while at the same time increasing the risk for obesity.

\section{Decreased concentrations of the lipoprotein lipase inhibitor angiopoietin-like protein 4 and increased serum triacylglycerol are associated with increased neonatal fat mass in pregnant women with gestational diabetes mellitus}

Ortega-Senovilla $H^{1}$, Schaefer-Graf $U^{2}$, Meitzner $K^{2}$, Abou-Dakn $M^{2}$, Herrera $E^{1}$

${ }^{1}$ Faculties of Pharmacy and Medicine, University CEU San Pablo, Madrid, Spain; and ${ }^{2}$ Department of Obstetrics and Gynecology,
Center for Diabetes in Pregnancy, St. Joseph's Hospital, Berlin, Germany

J Clin Endocrinol Metab: DOI: 10.1210/jc.2013-1614

\section{Objective}

Angiopoietin-like protein 4 (ANGPTL4) is an inhibitor of lipoprotein lipase (LPL) activity. Past studies have suggested that changes in LPL activity affect the transfer of fatty acids to the fetus and affect growth. This study aimed to evaluate the impact on neonatal fat mass (FM) by concentrations of ANGPTL4 and triacylglycerols (TAG) in maternal blood samples and umbilical cord blood of pregnant women with GDM.

\section{Methods}

Fasting maternal blood samples were drawn during pregnancy no longer than 1 week before delivery, and umbilical cord blood was taken after the vaginal delivery during 80 pregnancies complicated by GDM and 90 controls without GDM. All neonates were grouped based on FM for the 25th percentile or lower, 25 th to 75 th percentile, or 75 th to 100 th percentiles. Controls matched for age and pregestational BMI.

\section{Results}

The highest concentrations of TAG and nonesterified fatty acids (NEFA) were found in the maternal blood samples of women with GDM whose infants were born with the highest FM. These women also showed the lowest levels of ANGPTL4. Lower levels of ANGPTL4 were observed in both GDM cases and controls among infants born with the highest FM. Glucose and insulin concentrations were independent of any change to FM. Umbilical cord blood of infants born with the highest FM of women with GDM had higher concentrations of insulin and lower TAG levels than those with lower FM. There was no appreciable difference in NEFA or ANGPTL4 levels between GDM cases and controls.

\section{Conclusions}

Low levels of ANGPTL4 were associated with an increased placental transfer of lipids. This observation was found in both control cases and women with well-controlled GDM. Higher neonatal FM and TAG levels, along with a decreased ANGPTL4, were observed in pregnancies with well-controlled GDM. Increased maternal TAG was associated with a decreased TAG level in the umbilical cord blood. An increased transfer of lipids across the placenta would contribute to higher fetal FM. GDM pregnancies tended to have higher levels of insulin, and the effect of LPL may have been impacted by the hyperinsulinemia.

\section{Comment}

Because of the apparent effect of ANGPTL4 levels on neonatal birth weight, it may be worth considering this laboratory value in a clinical setting when assessing GDM patients. However, it is important to consider the relatively small sample size of this study. There may have been a trend simply among the group of patients, as all cases came from the same institution. Despite the sample size, the findings of the study appear valid and should be considered in future clinical care of GDM patients. 
1,5-Anhydroglucitol as a marker of maternal glycaemic control and predictor of neonatal birthweight in pregnancies complicated by type 1 diabetes mellitus

Nowak $N^{1}$, Skupien $J^{1,2}$, Cyganek $K^{3}$, Matejko $B^{1}$, Malecki $\mathrm{MT}^{1,3}$

${ }^{1}$ Department of Metabolic Diseases, Jagiellonian University Medical College, Krakow, Poland; ${ }^{2}$ Section on Genetics and Epidemiology, Joslin Diabetes Center, Boston, MA; and ${ }^{3}$ Department of Metabolic Diseases, University Hospital, Krakow, Poland

Diabetologia 2013; 56: 709-13

\section{Objective}

Despite reaching $\mathrm{HbA1c}$ goals, women with diabetes are still faced with an increased risk of macrosomic infants. This study aimed to measure the use of 1,5-anhydroglucitol $(1,5-$ AG) in monitoring glycemic control in pregnant women compared with the standard observation of $\mathrm{HbA1c}$ levels.

\section{Methods}

Eighty-two pregnant type 1 diabetic women were observed over the course of 4 years. Every trimester, their 1,5AG as well as $\mathrm{HbA1c}$ levels were monitored, and 58 of these women also had continuous glucose monitoring system (CGMS) data that were analyzed over a 7-day period before 1,5-AG and $\mathrm{HbA} 1 \mathrm{c}$ values were collected. CGMS data and birth weight were then analyzed against 1,5-AG and $\mathrm{HbA1c}$ levels using linear and logistic regression models, and receiver operating characteristic (ROC) analysis was used to model the association between third-trimester 1,5-AG levels and macrosomia. Macrosomia was defined as a birth weight over the 90 th percentile.

\section{Results}

Throughout the trial, the women met $\mathrm{HbA} 1 \mathrm{c}$ targets and maintained good glycemic control. HbA1c levels were not correlated with 1,5-AG levels; however, 1,5-AG levels were strongly correlated with CGMS mean glucose levels. 1,5-AG levels correlated with hyperglycemia, but not with hypoglycemia. About $80 \%$ of macro soma cases occurred in women with $\mathrm{HbA} 1 \mathrm{c}$ levels $<6.0 \%$. However, the average 1,5-AG levels in the second and third trimester were significantly lower in women with macrosomic infants, with third-trimester 1,5-AG levels as the stronger indicator of macrosomia. In the ROC analysis, the area under the curve resulted in a significantly better model for predicting birth weight when $\mathrm{HbA1c}$ and 1,5-AG levels were considered in tandem.

\section{Conclusions}

This study indicates that $1,5-\mathrm{AG}$ is a more valuable marker than $\mathrm{HbA1c}$ for observing episodes of hyperglycemia in pregnant women with type 1 diabetes. These values were strongly correlated to the CGMS data and were a stronger predictor of macrosomia than $\mathrm{HbA} 1 \mathrm{c}$ alone. Because 1,5-AG levels change rapidly with glucose levels, they may improve treatment and dosage modifications in pregnant type 1 diabetic patients. Third-trimester 1,5-AG levels were strongly associated with infant birth weights, and an even stronger model for predicting macrosomia resulted from a combined analysis of both the HbA1c and 1,5-AG levels.

\section{Comment}

Prevalence of macrosomia may be because of the HbA1c target itself $(6.0 \%)$ being too high and not attributed to the $\mathrm{HbA} 1 \mathrm{c}$ readings themselves. 1,5-AG appears to be a valid, useful marker of recent hyperglycemic levels. However, point-of-care $\mathrm{HbA} 1 \mathrm{c}$ is more practical in a primary care setting as it can be measured more immediately. Additionally, if $1,5-\mathrm{AG}$ is not associated with hypoglycemia, it may be dangerous to observe this value alone. In the future it may be valid to consider both $\mathrm{HbA} 1 \mathrm{c}$ and 1,5-AG levels in the second and third trimesters in order to predict macrosomia in the babies of type 1 diabetic women.

\section{A randomized trial comparing perinatal outcomes using insulin detemir or neutral protamine Hagedorn in type 1 diabetes}

Hod $M^{1}$, Mathiesen $E R^{2-4}$, Jovanovič $L^{5}$, McCance $D R^{6}$, Ivanisevic $M^{7}$, Durán-Garcia $S^{8}$, Brøndsted $L^{9}$, Nazeri $A^{9}$, $\operatorname{Damm} P^{2,4,10}$

${ }^{1}$ Helen Schneider Women's Hospital, Rabin Medical Center, Sackler Faculty of Medicine, Tel Aviv University, Tel Aviv, Israel; ${ }^{2}$ Center for Pregnant Women with Diabetes, Rigshospitalet, ${ }^{3}$ Department of Endocrinology, and ${ }^{4}$ Faculty of Health Sciences, University of Copenhagen, Copenhagen, Denmark; ${ }^{5}$ Sansum Diabetes Research Institute, Santa Barbara, CA; ${ }^{6}$ Metabolic Unit, Royal Victoria Hospital, Belfast, United Kingdom; ${ }^{7}$ Department of Obstetrics and Gynecology, University Hospital of Zagreb, Zagreb, Croatia; ${ }^{8}$ Catedra de Endocrinologia, Unidad de Diabetes y Embarazo, Hospital Universitario de Valme, Seville, Spain; ${ }^{9}$ Novo Nordisk A/S, Søborg, Denmark; and ${ }^{10}$ Department of Obstetrics, Rigshospitalet, University of Copenhagen, Copenhagen, Denmark

J Matern Fetal Neonatal Med 2013 June 5: [Epub ahead of print]

\section{Maternal efficacy and safety outcomes in a randomized, controlled trial comparing insulin detemir with NPH insulin in $\mathbf{3 1 0}$ pregnant women with type 1 diabetes}

Mathiesen $E^{1-3}$, Hod $M^{4}$, Ivanisevic $M^{5}$, Duran Garcia $S^{6}$, Brøndsted $L^{7}$, Jovanovic $L^{8}$, Damm $P^{1,3,9}$, McCance $D R^{10}$; on behalf of the Detemir in Pregnancy Study Group

${ }^{1}$ Center for Pregnant Women with Diabetes, Rigshospitalet, ${ }^{2}$ Department of Endocrinology, and ${ }^{3}$ Faculty of Health Sciences, University of Copenhagen, Copenhagen, Denmark; ${ }^{4}$ Helen Schneider Women's Hospital, Rabin Medical Centre, Sackler Faculty of Medicine, Tel Aviv University, Tel Aviv, Israel; ${ }^{5}$ Department of Obstetrics and Gynecology, University Hospital of Zagreb, Zagreb, Croatia; ${ }^{6}$ Catedra de Endocrinologia, Unidad de Diabetes y Embarazo, Hospital Universitario de Valme, Seville, Spain; ${ }^{7}$ Novo Nordisk A/S, Søborg, Denmark; ${ }^{8}$ Sansum Diabetes Research Institute, Santa Barbara, CA; ${ }^{9}$ Department of Obstetrics, Rigshospitalet, University of Copenhagen, Copenhagen, Denmark; and ${ }^{10}$ Metabolic Unit, Royal Victoria Hospital, Belfast, United Kingdom

Diabetes Care 2012; 35: 2012-17 


\section{Objective}

In nonpregnant diabetic patients, short- and long-acting insulin analogs have been shown to be more effective than human insulins at facilitating healthy glycemic control; however, few studies have tested the use of long-acting insulin analogs in pregnant women with type 1 diabetes. This is the first clinical trial to compare the safety and efficacy of the basal insulin analog, detemir (IDet), with human neutral protamine Hagedorn (NPH), both in combination with rapid-acting insulin aspart (IAsp), in pregnant women with type 1 diabetes. The publication by Hod et al. reports the perinatal and obstetric outcomes, while a separate article by Mathiesen et al. reports the maternal outcomes of the pregnant women in the same trial.

\section{Methods}

This was a randomized, controlled noninferiority trial involving 310 women with type 1 diabetes conducted at 79 sites in 17 countries. The women were either randomized to IDet $(49.0 \%)$ or to NPH (51.0\%). The primary endpoint analyzed was $\mathrm{HbA} 1 \mathrm{c}$ at 36 gestational weeks as an indicator of glycemic control. Perinatal outcomes across the two treatment arms, reported in Hod et al., were analyzed in terms of three main categories: fetal death, termination of pregnancy, and live birth. The composite pregnancy outcome was defined as the incidence of at least one of the following secondary outcomes: small/large ( $<10$ th or $>90$ th percentile) for gestational age, early fetal death, perinatal or neonatal mortality, congenital malformations, and/or preterm delivery. Maternal efficacy endpoints, as described in Mathiesen et al., included $\mathrm{HbA1c}$ values at $8-12,14$, and 24 gestational weeks, fasting plasma glucose (FPG) levels, number of hypoglycemic episodes, insulin dose, and weight gain during pregnancy.

\section{Results}

The primary endpoint, estimated mean $\mathrm{HbA} 1 \mathrm{c}$ at 36 gestational weeks, did not statistically differ across the IDet and $\mathrm{NPH}$ treatment arms. The composite pregnancy outcomes detailed in Hod et al. were comparable between the two groups. Gestational age at delivery was significantly greater for the offspring of women treated with IDet compared with those of women treated with NPH. No other statistically significant differences in perinatal outcomes were found between the two treatment groups. As described in Mathiesen et al., estimated mean FPG levels were significantly lower in IDet patients compared with NPH patients at both 24 and 36 gestational weeks. No statistically significant differences were observed with regard to weight gain, insulin dose, or number of hypoglycemic episodes between the two groups.

\section{Conclusions}

This study demonstrates that the use of IDet during pregnancy is safe and effective with respect to both maternal and perinatal/obstetric outcomes, and is noninferior to NPH for glycemic control in type 1 diabetic mothers. Additionally, maternal FPG levels are significantly lower in IDet-treated subjects compared with NPH-treated subjects, without an accompanying increase in the number of hypoglycemic episodes. A larger sample size is required to analyze the occurrence of uncommon perinatal outcomes with statistical significance.

\section{Comment}

IDet features an advantage over NPH in its convenience of administration; IDet has a mean lifetime of 12 hours within the patient and can be injected twice daily, while $\mathrm{NPH}$ has a mean lifetime of 8 hours and must be taken three times to account for a 24-hour period. We note, however, that in this trial, basal insulin was administered once or twice daily across both treatment arms. This method would favor optimal FPG levels in the IDet group because of inadequate insulin dosing in the NPH group. Further studies may confirm whether IDet truly facilitates lower FPG levels in comparison to NPH insulin that is administered three times daily.

\section{Insulin resistance and impaired pancreatic beta-cell function in adult offspring of women with diabetes in pregnancy}

Kelstrup $L^{1,2,4}$, Damm $P^{1,2,5}$, Mathiesen $E R^{1,3,5}$, Hansen $T^{6,7}$, Vaag $A A^{3,5,8}$, Pedersen $\mathrm{O}^{5,6,9}$, Clausen $\mathrm{TD}^{1,2,10}$

${ }^{1}$ Center for Pregnant Women with Diabetes, Rigshospitalet, University of Copenhagen, Copenhagen, Denmark; Departments of ${ }^{2}$ Obstetrics and ${ }^{3}$ Endocrinology, University of Copenhagen, Copenhagen, Denmark; ${ }^{4}$ Danish PhD School of Molecular Metabolism, Odense C, Denmark; ${ }^{5}$ Faculty of Health Science, University of Copenhagen, Denmark; ${ }^{6}$ Novo Nordisk Foundation Center for Basic Metabolic Research, Metabolics Genetics, Faculty of Health Science, University of Copenhagen, Denmark; ${ }^{7}$ Faculty of Health Sciences, University of Southern Denmark, Denmark; ${ }^{8}$ Diabetes and Metabolism, Department of Endocrinology, Rigshospitalet, Copenhagen, Denmark; ${ }^{9}$ Faculty of Health Sciences, University of Aarhus, Aarhus, Denmark; and ${ }^{10}$ Department of Obstetrics and Gynecology, Hilleroed Hospital, University of Copenhagen, Hilleroed, Denmark

J Clin Endocrinol Metab 2013; 98: 3793-801

\section{Objective}

It is known that maternal hyperglycemia during pregnancy puts offspring at greater risk for metabolic diseases later in life. The purpose of this study was to examine the effects of either GDM or type 1 diabetes in pregnant Caucasian women on insulin sensitivity and/or insulin release in their adult offspring.

\section{Methods}

In this prospective cohort study, 597 individuals born between 1978 and 1985 were followed up between the ages of 18 and 25 and assessed for insulin sensitivity, absolute insulin release, and disposition index (a measure of insulin release that factors into a hyperbolic relationship with insulin sensitivity). Exposed adult offspring were organized into 2 groups based on their mode of exposure to intrauterine hyperglycemia: offspring of women with GDM during pregnancy and offspring of women with type 1 diabetes. Also included were 2 reference groups unexposed to intrauterine hyperglycemia: the offspring of non-GDM women at high risk for developing GDM, and the offspring of women of the background population, at low risk for developing GDM. 


\section{Results}

Offspring of women with GDM during pregnancy (O-GDM), of women with type 1 diabetes during pregnancy (O-Type1), and of nondiabetic women at higher risk for developing GDM (O-NoGDM) all had significantly reduced insulin sensitivities compared with the offspring of women at low risk for developing GDM (O-BP). Similar trends were observed regarding reduced disposition index in the O-GDM, O-Type1, and O-NoGDM groups in comparison to the O-BP group, although there were no significant differences in absolute insulin response between the four groups.

\section{Conclusions}

The adult offspring of women with either type 1 or gestational diabetes present with lower insulin sensitivity and lower insulin response than their counterparts with no maternal risk factors. Impaired insulin action and secretion puts such offspring at greater risk for developing type 2 diabetes, although further studies are needed in order to evaluate the overall risk when compounded with other factors.

\section{Comment}

While intrauterine hyperglycemia appears to put offspring at risk for developing impaired insulin action and secretion levels, it is also noteworthy that the O-NoGDM subjects, whose mothers were not diabetic but were at risk for developing GDM, demonstrated insulin sensitivity and insulin response levels similar to their counterparts in the two exposed groups. These results suggest that genetic predisposition may play as significant a role as exposure to intrauterine hyperglycemia in promoting insulin resistance and impaired pancreatic $\beta$-cell function. Nonetheless, the authors note that the mothers of the O-NoGDM subjects demonstrated slightly higher glucose levels than the mothers of the O-BP subjects, so intrauterine hyperglycemia cannot yet be discounted as a risk factor until further studies are conducted. Pregnant women with GDM are encouraged to continue maintaining healthy glycemic control to prevent metabolic diseases and other health problems in their offspring later in life.

\section{Comparison of insulin lispro protamine suspension with NPH insulin in pregnant women with type 2 and gestational diabetes mellitus: maternal and perinatal outcomes}

\author{
Colatrella $A^{1}$, Visalli $N^{2}$, Abbruzzese $S^{2}$, Leotta $S^{2}$, \\ Bongiovanni $M^{1}$, Napoli $A^{1}$ \\ ${ }^{1}$ Department of Clinical and Molecular Medicine, S. Andrea \\ Hospital, Faculty of Medicine and Psychology, Sapienza Uni- \\ versity, Rome, Italy; and ${ }^{2}$ Unit of Dietology, Diabetology and \\ Metabolic Diseases, Sandro Pertini Hospital, Rome, Italy
}

Int J Endocrinol 2013; 2013: Article ID 151975, 8 pages

\section{Objective}

While rapid-acting insulin analogs such as lispro and aspart are now widely used, human NPH remains the primary basal insulin for pregnant diabetic women. Insulin lispro protamine suspension (ILPS), an intermediate-acting basal insulin analog, shows promise as a valuable option for glycemic control; however, no studies regarding its use during pregnancy were performed prior to this study. This study aimed to demonstrate the efficacy of ILPS in facilitating positive maternal and perinatal outcomes in pregnant women with type 2 and gestational diabetes.

\section{Methods}

This study was a retrospective cohort study of 89 pregnant women with type 2 or GDM recruited from January 2008 to August 2010 in two hospitals in Rome. In addition to medical nutrition therapy and short-acting insulin analogs, subjects were treated with either ILPS $(60 \%)$ or $\mathrm{NPH}(40 \%)$ as basal insulins. Outcomes measured included glycemic control, insulin dose, hypoglycemic episodes, hypertension, and mode of delivery.

\section{Results}

Maternal outcomes, such as glycemic control, rates of hypertension, and number of hypoglycemic episodes, were comparable between the ILPS and NPH treatment groups. Differences were observed in the ponderal indices of newborns, as subjects from the NPH treatment group gave birth to 3 newborns with a ponderal index of $>2.85 \%$, compared with zero subjects in the ILPS treatment group. Additionally, while subjects in both groups used similar doses of intermediateacting insulin, ILPS-treated women required smaller total dosages of insulin than their NPH-treated counterparts.

\section{Conclusions}

The use of either ILPS or NPH intermediate-acting insulin in pregnant women with type 2 or gestational diabetes leads to comparable maternal and pregnancy outcomes, with the exception of a greater prevalence of high-ponderal-index infants and greater total insulin dosages for NPH-treated women. The use of either insulin during pregnancy seems safe.

\section{Comment}

This study demonstrates that the efficacy of ILPS is comparable to that of NPH. According to pharmacokinetic and glucodynamics studies, ILPS may also feature a longer duration of action than NPH, similar to detemir and glargine, reducing the total insulin dosage requirement. Because insulin analogs can potentially interact with IGF-1 or insulin receptors in different ways from human insulin, it is noteworthy that ILPS presents a possible advantage over NPH in preventing LGA infants; however, larger studies are needed to confirm these effects.

The effect of maternal obesity on pregnancy outcomes of women with gestational diabetes controlled with diet only, glyburide, or insulin

Joy $S^{1}$, Roman $A^{2}$, Istwan $N^{3}$, Rhea $D^{3}$, Desch $C^{3}$, Stanziano $G^{3}$, Saltzman $D^{2}$ 
${ }^{1}$ Carolinas Medical Center, Charlotte, NC; ${ }^{2}$ Maternal Fetal Medicine Associates, PLLC, New York, NY; and ${ }^{3}$ Alere Health, Women's and Children's Health, Department of Clinical Research, Atlanta, GA

Am J Perinatol 2012; 29: 643-48

\section{Objective}

The purpose of this study was to examine the effects of maternal obesity in women with GDM on pregnancy and neonatal outcomes. Additionally, the authors compared the effectiveness of diet-only, glyburide, and insulin treatments in controlling GDM across obese and nonobese populations.

\section{Methods}

In this retrospective cohort study, the data of 7,020 women with GDM during singleton pregnancies were collected and analyzed for maternal characteristics and pregnancy outcomes. The outcomes for obese versus nonobese women were evaluated across three treatment groups: diet-only, glyburide, and insulin. Logistic regression analysis was employed to compare the efficacy of the three treatment plans independent of maternal characteristics.

\section{Results}

Across all three treatment groups, obese women were more likely to develop pre-eclampsia, have a cesarean delivery, or give birth to infants with macrosomia. Furthermore, logistic regression results indicate that the newborn offspring of women treated with glyburide were more than two times as likely to develop hyperbilirubinemia. Glyburide treatment was also associated with a greater risk of preeclampsia in GDM patients, even after correcting for obesity and parity.

\section{Conclusions}

This study provides evidence to support that obese mothers with GDM are at a greater risk for adverse maternal and perinatal outcomes in comparison to their nonobese counterparts, even when obese mothers achieve safe glycemic levels. In addition, preliminary results suggest that the use of glyburide during pregnancy is unsafe for both the mother and the fetus, with heightened risks of pre-eclampsia and hyperbilirubinemia. Further randomized controlled studies are needed in order to directly examine the risks associated with glyburide use for glycemic control during pregnancy.

\section{Comment}

Potential adverse outcomes may be associated with glyburide use, including a greater incidence of fetal macrosomia. Although this study was not designed to compare the efficacy of glyburide and insulin in GDM patients, the results regarding elevated risks for pre-eclampsia and hyperbilirubinemia call for further caution when moving away from insulin as the primary treatment for gestational diabetes. In addition to the suggested adverse physiological effects, GDM women receiving glyburide treatment are less likely to monitor blood glucose levels meticulously because of the association of lower-risk cases of GDM with oral hypoglycemic agents, in contrast to injected insulin treatments. In summary, glyburide is not recommended for use in women with GDM until definitive results are collected regarding its safety and efficacy during pregnancy, particularly concerning potential transplacental transfer. It is also critical that all women found to have GDM strive to maintain healthy glycemic control and body weight in order to achieve optimal pregnancy outcomes.

\section{The effect of real-time continuous glucose monitoring in pregnant women with diabetes: a randomized controlled trial}

Secher $A L^{1-3}$, Ringholm $L^{1,2}$, Andersen $H U^{4}$, Damm $P^{1,3,5}$, Mathiesen $E^{1-3}$

${ }^{1}$ Center for Pregnant Women with Diabetes, Rigshospitalet, Copenhagen, Denmark; ${ }^{2}$ Department of Endocrinology, Rigshospitalet, Copenhagen, Denmark; ${ }^{3}$ Faculty of Health Sciences, University of Copenhagen, Denmark; ${ }^{4}$ Steno Diabetes Center, Gentofte, Denmark; and ${ }^{5}$ Department of Obstetrics, Rigshospitalet, Copenhagen, Denmark

Diabetes Care 2013; 36: 1877-83

\section{Objective}

Pregnant women with type 1 or type 2 diabetes are at greater risk for maternal hyper- and hypoglycemia and adverse pregnancy outcomes, such as LGA infants. The purpose of this study was to determine whether intermittent real-time continuous glucose monitoring (CGM) facilitates superior glycemic control for women with diabetes in comparison to the traditional method of self-monitored plasma glucose (SMPG) several times daily.

\section{Methods}

This was a randomized controlled trial involving 123 women with type 1 diabetes and 31 women with type 2 diabetes from February 2009 to February 2011, all of whom accepted participation in the study before completing the 14th week of gestation. While all women in the study received routine pregnancy care, 79 women $(51 \%)$ were also randomized to intermittent use of real-time CGM. Results on HbA1c values and insulin doses were recorded during five antenatal clinic visits at $8,12,21,27$, and 33 gestational weeks. The primary outcome of the study was defined as the prevalence of LGA infants, and the secondary combined endpoint was defined as the prevalence of preterm delivery and/or severe neonatal hypoglycemia.

\section{Results}

The primary outcome, the prevalence of LGA infants, was comparable between the two treatment arms $(p=0.19)$, as was the secondary combined outcome, the prevalence of preterm delivery and/or severe neonatal hypoglycemia $(p=0.36)$. Maternal glycemic control, gauged by HbA1c levels, SMPG values, and hypoglycemic events, was also similar between the CGM and control groups. 


\section{Conclusions}

No statistically significant differences were observed in maternal glycemic control or pregnancy outcomes between subjects using real-time CGMs and control subjects receiving only routine pregnancy care. According to the results of this trial, the use of CGMs by pregnant women with pregestational diabetes provides no discernible advantages over SMPG.

\section{Comment}

As real-time CGM technology is significantly costlier than SMPG, it is gratifying to note that traditional fingersticks can be just as effective as CGMs at facilitating glycemic control in diabetic pregnant mothers. This study serves as a reminder that medical nutrition therapy and lifestyle modification have the greatest impact on positive pregnancy outcomes. However, it is also noteworthy that very few women in this trial were willing to use real-time CGM continuously, which may have contributed to the lack of significant differences in outcome. It will be interesting to see in future studies how real-time CGMs may benefit patients with higher baseline $\mathrm{HbA1c}$ levels than those included in this trial.

\section{Treatment with the long-acting insulin analogues detemir or glargine during pregnancy in women with type 1 diabetes: comparison of glycaemic control and pregnancy outcome}

Callesen $N F^{1,2}$, Damm $J^{1,2}$, Mathiesen $J M^{1,2}$, Ringholm $L^{1,2}$, Damm $P^{1,3,4}$, Mathiesen $E R^{1-3}$

${ }^{1}$ Center for Pregnant Women with Diabetes, Rigshospitalet, Copenhagen, Denmark; ${ }^{2}$ Department of Endocrinology, Rigshospitalet, Copenhagen, Denmark; ${ }^{3}$ Faculty of Health Sciences, University of Copenhagen, Denmark; and ${ }^{4}$ Department of Obstetrics, Rigshospitalet, Copenhagen, Denmark

J Matern Fetal Neonatal Med 2013; 26: 588-92

\section{Objective}

Long-acting insulin analogs are becoming increasingly popular for glycemic control in nonpregnant diabetic patients, as they are associated with a lower incidence of nocturnal hypoglycemia than human insulins. However, few studies have been conducted to investigate the use of these analogs in pregnancies complicated by type 1 diabetes. This is the first study to compare glycemic control and pregnancy outcomes in type 1 diabetic women between two such long-acting insulin analogs, detemir and glargine.

\section{Methods}

This was a retrospective cohort study conducted at a single diabetes clinic in Rigshospitalet, Copenhagen, Denmark, from January 2007 to August 2011. Of the 113 women involved in the study, 67 received insulin detemir and 46 received glargine from conception to delivery. Subjects were evaluated based on maternal outcomes such as $\mathrm{HbA1c}$ levels at 8 and 33 weeks, SMPG values 7 times daily, number of hypoglycemic events, number of daily injections, and total insulin dose. Perinatal outcomes, such as rates of neonatal morbidity and LGA (>90th percentile) infants, were also reported.

\section{Results}

Many outcomes, such as HbA1c levels and rates of hyperglycemia, were comparable between the two treatment arms. However, women treated with detemir were administered larger doses of long-acting insulin at 8 and 33 weeks, and were also more likely to receive 2 or more daily injections of long-acting insulin at 8 and 33 weeks. Furthermore, a greater incidence of LGA infants was observed in the detemir treatment group $(p=0.046)$. Both treatment groups experienced low incidences of congenital malformations, and no perinatal deaths were reported.

\section{Conclusions}

Glycemic control and pregnancy outcomes are comparable in women treated with the insulin analogs detemir and glargine, with low rates of maternal and perinatal adverse events in both groups. Larger studies are needed to confirm the higher prevalence of LGA infants in women treated with detemir.

\section{Comment}

Some of the different outcomes between the two treatment arms in this study may not be attributed to the insulin analogs used by the subjects, but rather because of poor glycemic control; mean $\mathrm{HbA1c}$ levels were high in both groups at 8 and 33 weeks of gestation, ranging from $6.1 \%$ to $6.8 \%$. Additionally, the finding that a greater number of women in the detemir group required two or more daily injections of long-acting insulin is not surprising, given that detemir features a shorter duration of action (12 hours) in comparison to glargine (18-30 hours). Inadequate doses of long-acting insulin and a greater proportion of gravida 1 mothers $(p=0.02)$ may have been factors leading to a greater incidence of LGA infants in the detemir treatment arm.

\section{Global methylation in the placenta and umbilical cord blood from pregnancies with maternal gestational diabetes, preeclampsia, and obesity}

Nomura $Y^{1,2}$, Lambertini $L^{3,4}$, Rialdi $A^{3}$, Lee $M^{4}$, Mystal $E Y^{1,2}$, Grabie $M^{1,2}$, Manaster $I^{2}$, Huynh $N^{1,2}$, Finik $J^{1,2}$, Davey $M^{2}$, Davey $K^{2}$, Ly $J^{1,2}$, Stone $J^{5}$, Loudon $H^{5}$, Eglinton $G^{6}$, Hurd $Y^{2,7}$, Newcorn $J^{2,8}$, Chen $J^{3,8,9}$

${ }^{1}$ Department of Psychology, Queens College, CUNY, Flushing, $N Y$; Departments of ${ }^{2}$ Psychiatry, ${ }^{3}$ Preventive Medicine, ${ }^{5} \mathrm{Ob}$ stetrics, Gynecology, and Reproductive Science, ${ }^{8}$ Pediatrics, and ${ }^{9}$ Oncological Sciences, Mount Sinai School of Medicine, New York, NY; ${ }^{4}$ Department of Obstetrics and Gynecology, Indiana University, Indianapolis, IN; ${ }^{6}$ Department of Obstetrics and Gynecology, Weill Medical College of Cornell University, Flushing, NY; and ${ }^{7}$ James J. Peters VA Medical Center, Bronx, NY

Reprod Sci 2013 June 13: [Epub ahead of print]: DOI: 10.1177/ 1933719113492206 


\section{Objective}

Epigenetic modifications to the genome during early development can cause permanent changes in gene expression and physiological processes. It has also been observed that nonideal maternal conditions, such as GDM, pre-eclampsia, and obesity, are risk factors for future diseases in the offspring. The purpose of this study was to investigate the link between global methylation levels in the placenta and umbilical cord blood, and maternal risk factors such as gestational diabetes, pre-eclampsia, and obesity.

\section{Methods}

In a pilot study at a low-income clinic in New York City, 50 women were followed from the second trimester of pregnancy to delivery. All subjects were screened for GDM, pre-eclampsia, and obesity during pregnancy. Following delivery, matched samples of placenta and umbilical cord blood were collected for DNA/RNA extraction, and global methylation levels were measured with the Lumino-metric Methylation Assay. Multivariable analyses with a general linear model were conducted to examine the associations between global methylation levels, maternal risk factors, and neonatal outcomes.

\section{Results}

No associations were observed with global methylation levels in the umbilical cord blood and any of the three maternal risk conditions under study (GDM, pre-eclampsia, and obesity), or with any of the four neonatal outcomes (birth weight, gestational age, head circumference, and body length). However, women with GDM or pre-eclampsia had lower levels of global methylation in the placenta tissue, and women with obesity had higher levels of global methylation in the placenta tissue in comparison to their lower-risk counterparts. In addition, a suggestive correlation was found between higher levels of global methylation in the placenta and reduction in infant body length and head circumference, although these results did not attain statistical significance.

\section{Conclusions}

This study presents preliminary evidence that maternal risk conditions during pregnancy, such as GDM, pre-eclampsia, and obesity, influence global methylation levels in the placenta, which may in turn cause adverse changes in fetal growth in the offspring.

\section{Comment}

The emerging field of epigenetics provides a biological mechanism for the propagation of health risk factors from pregnant women to offspring. It will be exciting to see the results of future studies that further investigate the developmental trajectories of these offspring into childhood and adolescence. However, no definitive conclusions about global methylation levels in the placenta can be made without larger sample sizes and a more diverse cohort of subjects with regard to ethnicity, living environment, and socioeconomic background.

\section{Conclusion}

In summary, research on diabetic complications of pregnancy has expanded significantly over the past year. It was more difficult than ever to narrow down our selection of articles, with over 1,200 relevant publications on the subject. Hyperglycemia has the potential to lead to a variety of negative outcomes for both the mother and child, and so this renewed interest in the field will have a tangible impact on patients affected by diabetes. During pregnancy, many women will be willing to make lifestyle changes for the benefit of their baby. Providers should strive not only to treat maternal hyperglycemia, but also to provide health education that will enable the patient to understand the importance of controlling the disease. The explosion of varied research is exciting, and will surely drive future studies to promote a stronger understanding of maternal hyperglycemia, improving the care and outcomes of pregnancies complicated by diabetes.

\section{Author Disclosure Statement}

No competing financial interests exist.

\section{Reference}

1. Diabetes Statistics. Data from the 2011 National Diabetes Fact Sheet (released Jan. 26, 2011). Available online at www .diabetes.org/diabetes-basics/diabetes-statistics/ 